\title{
Vous allez dire que ma lettre n'est pas encourageante. Que voulez-vous, je suis franc et j'ai tenu à vous dire tout cela... Ou comment les poilus réduisent la « distance » dans leurs lettres
}

\author{
Vicari, Stefano \\ Università degli Studi di Genova \\ stefano.vicari82@gmail.com
}

\section{Introduction}

Ainsi que de nombreux historiens l'ont déjà remarqué, la Grande Guerre a représenté une véritable ligne de partage sous plusieurs points de vue: la mobilisation de masse, les nouveaux moyens techniques utilisés, la durée et les dimensions du conflit mondial, etc. Il n'est donc pas étonnant que les soldats, une fois que la guerre se stabilise dans les tranchées sur plusieurs fronts, ressentent le besoin de renouer les contacts avec l'arrière par l'écriture. En effet, en France, près de 10 milliards de lettres (4 millions par jour) ont été envoyées par les poilus, pendant les quatre années de guerre. ${ }^{1}$ C'est aussi pour cette raison que, ces dernières décennies, les historiens ${ }^{2}$ ont commencé à montrer un intérêt croissant pour ces lettres, qui représentent des sources historiographiques d'une richesse et d'une importance fondamentales pour répondre à toute une série de questions, aujourd'hui encore sans réponses définitives, comme par exemple : comment expliquer la mobilisation massive à la guerre ? Jusqu'où s'étend le consentement des soldats à la mobilisation et, plus en général, à la guerre ?

Sans prétendre répondre exhaustivement à ces questions, je me propose dans cette étude de montrer comment les outils de l'analyse du discours peuvent nous aider à mettre en lumière l'inscription en discours d'une dimension argumentative (Amossy, 2010 [2000]) plus ou moins implicite et, par là même, de contribuer à cerner les raisons pour lesquelles ce phénomène d'écriture populaire a pris de si amples dimensions.

Après avoir précisé ce que j'entends par réduire la distance dans les lettres des poilus et après avoir présenté mes hypothèses, je montrerai le rôle des lettres pour les soldats au front à partir de leurs propres commentaires : cette première partie me permettra de rendre compte du contexte au sens large où se déroulent ces échanges épistolaires et de montrer les enjeux de ce type de correspondances. Ensuite, j'essayerai de répondre à la question de savoir comment les mots des poilus réduisent la distance imposée par les contraintes physiques et par l'adoption du genre de la correspondance épistolaire.

\section{Que signifie réduire la distance dans le contexte des lettres des poilus?}

\subsection{Distances physique, symbolique et distance énonciative: quelles articulations?}

Tout échange épistolaire repose sur l'absence physique de l'autre et sur sa présence en discours comme les études en linguistique textuelle (Adam, 1997), en linguistique interactionnelle (Kerbrat-Orecchioni, 1998) et en pragmatique (Jaubert, 2005) l'ont déjà souligné : la conditio sine qua non pour qu'une correspondance épistolaire ait lieu réside justement dans la distance physique qui empêche aux deux locuteurs de mener une conversation en face à face, du moins pour les échanges authentiques qui font l'objet de cette étude. Il s'agit d'une distance géographique, qui ne permet pas aux destinataires de vivre 
certaines situations vécues par les soldats comme extraordinaires, bref de partager la meme situation d'énonciation.

Ce n'est pourtant pas la seule distance à laquelle le titre de cette contribution se réfère. A côté des contraintes physiques et objectives, en effet, il me semble que les lettres des poilus se caractérisent par la mise en discours d'une distance pourrait-on dire « subjective », symbolique, dénoncée par les scripteurs lorsqu'ils se rendent compte du décalage entre les discours autres, « légitimes », officiels et délivrés par la presse, auxquels sont exposés leurs proches à l'arrière et leur expérience au front et à leurs propres mots pour la décrire.

De par la dénonciation de ce second type de distance, les lettres deviendraient l'outil permettant aux soldats de transmettre à leurs familles ce qu'ils considèrent comme le vrai discours sur la guerre et sur leur vécu, en réduisant ainsi la distance symbolique entre les scripteurs et leurs destinataires. Or, tantôt à cause de la présence massive de la censure, surtout à partir de $1915^{3}$, tantôt en raison de l'« indicibilité » de l'expérience telle qu'elle est exprimée à maintes reprises par les soldats, cette prise de distance ne peut se faire que de manière subreptice, quasi silencieuse. Elle se glisse dans les non-dits, dans les plis du discours et n'est repérable qu'à partir des traces d'un discours de contestation de la guerre et des conditions de vie qui leur sont imposées par celle-ci.

Du premier type de distance découle le second : comme l'affirme Kerbrat-Orecchioni « on écrit parce qu'on est séparés, en même temps que pour créer l'illusion qu'on est ensemble » (Kerbrat-Orecchioni 1998 : 17, ital. de l'auteure). Les dénominations données à la correspondance proposées par les études sur le genre épistolaire montrent pourtant clairement les ressemblances entre les deux types de communication : c'est ainsi que l'on retrouve des formules du type " dialogue à distance », « « une moitié de dialogue » (Jaubert, $2005: 216$ ), « dialogue épistolaire » (ibid., 218), « substituts de dialogue » (ibid., 226), etc. qui rendent compte d'une certaine filiation des deux genres.

Les lettres, écrites pour la plupart par des peu-lettrés, constituent un cas sui generis, puisque la dette envers l'oralité est évidente sous plusieurs points de vue : à partir d'une orthographe fortement oralisée, pour passer ensuite à l'emploi plus ou moins massif de mots argotiques et d'expressions populaires ou régionales colorées, pour arriver, enfin, à une syntaxe puisant fortement dans les constructions typiques de l'oral, seule manière de s'exprimer que la plupart des soldats utilisaient avant la guerre. Tous ces éléments concourent à créer un effet d'oralité.

Si ces affirmations sont vraies pour un grand nombre de correspondances (ce qui est montré par le fait que de nombreux recueils de lettres ont subi une révision linguistique avant d'etre publiés, comme l'affirment les auteurs dans les préfaces), dans les lettres que j'ai analysées, la place de l'écriture oralisée n'est pas préponderante ou, du moins, ne peut pas etre observée ${ }^{4}$. Le seul scripteur où certains phénomènes apparaissent avec une certaine constance est Maurice Papillon, le moins lettré parmi les auteurs des correspondances prises en compte et dont le recueil n'a subi aucune correction de la part des éditeurs au moment de la publication.Voici deux exemples illustratifs :

\author{
je vais etre vassiné aujourdhui pour 2 fois. [...] Esercisse n'est pas trop dur : on fait 2 \\ heures d'ésercisse par jours et 3 heures de dhéorie. J'ai touché le prê hier [...]. On a bu \\ un litre de cidre ensamble ${ }^{5}$. (Papillon M., le 12/01/1915, pp. 72-73). \\ Je ne suis été malade la $3^{\text {ème }}$ fois, mais la deusième fois, j'ai eu du mal au bras pandan \\ trois jours (Papillon M., le 02/01/1914, p. 78).
}

C'est une écriture qu'on pourrait définir «de paroles» que ses caractéristiques syntaxiques, orthographiques et lexicales rapprochent de très près d'une conversation en face à face (Branca-Rosoff, 1990). Au-delà d'une écriture redevable de l'oral, en effet, sous la plume des scripteurs eux-mêmes, l'on retrouve volontiers des comparaisons entre correspondance écrite et conversation :

Pour moi, ce ne sont pas des lettres, c'est de la conversation. Je vous raconte n'importe quoi, au petit bonheur, à tort à travers, comme on parle. Je promène mon papier, je le mets dans ma poche et, ce que je ne puis écrire, je le continue en imagination. Ça m'occupe, je pense à ce que me dit l'un de vous comme si j'allais répondre de vive voix. Il me semble que nous sommes ensemble. Aussi, quand les 
circonstances matérielles, en particulier la fatigue des tranchées ou les petites âneries dont D.T. [Dérange Tout, l'adjudant chef de son bataillon] amuse ses hommes viennent m'enlever la liberté d'esprit, ça ne va plus. Et c'est un besoin de plus en plus impétueux pour moi de m'abstraire ainsi d'une vie matériellement, intellectuellement et moralement dégoutante, déprimante, abrutissante. (Tanty E., pp. 203-204).

Cet extrait montre bien que l'explicitation de la ressemblance entre les deux genres est fonctionnelle à l'inscription en discours d'une critique du présent de la vie dans la tranchée qui s'appuie sur une dimension argumentative fondée, dans ce cas, sur le climax d'émotions dysphoriques que le lecteur partagera aisément.

Si les caractéristiques plus proprement langagières ne pourront pas être mises au crible de l'analyse, du point de vue discursif, ces lettres apparaissent comme prises dans une véritable jungle de discours rapportés, d'allusions à des discours autres ou déjà dits (Authier-Revuz, 1995), des silences dits et montrés qui en font un observatoire précieux de phénomènes pouvant etre appréhendés par le biais de catégories empruntées au cadre indiciel et pragmatique de l'énonciation et, notamment, à partir de la notion de distance énonciative.

Les scripteurs manifestent en effet différents degrés de prise en charge de leurs énoncés dans le but de réduire cette double distance géographique et symbolique qu'eux-mêmes thématisent souvent dans les lettres. En particulier, cette notion me paraît utile afin d'appréhender des phénomènes ayant trait au rejet du moins partiel de certaines voix convoquées dans les lettres par les scripteurs et qui relèvent de cas affichant une double nature dialogale et dialogique, comme j'essayerai de le montrer dans les analyses suivantes.

\subsection{Dialogal et dialogique : remarques théoriques, méthodologiques et intérêt de l'analyse}

Avant d'entrer dans le détail de la démarche théorique et méthodologique, quelques repères terminologiques sont nécessaires. Dans le sillage des ouvrages dictionnairiques de l'analyse du discours (Mainguenau et Charaudeau, 2002, Détrie, Siblot, Verine, 2001), pour les analyses qui suivent, j'adopterai la distinction entre dialogal et dialogique résumée par Bres de la manière suivante :

\footnotetext{
- dialogal, pour prendre en charge tout ce qui a trait au dialogue en tant qu'alternance de tours de parole, disons le dialogue externe pour parler comme Bakhtine [...] ; dialogal est opposé à monologal ;

- dialogique, pour prendre en charge la problématique de l'orientation de l'énoncé vers d'autres énoncés, disons pour faire vite le dialogue interne; dialogique est opposé à monologique. (Bres, 2005 : 99, ital. de l'auteur)
}

Il me semble en effet que les deux notions ainsi définies conviennent bien à l'analyse de ces lettres qui se configurent comme des lieux de tensions discursives diverses qui peuvent être artificiellement distinguées entre celles qui actualisent des relations interlocutives, donc d'origine dialogale, entre destinateur et destinataire et celles qui projettent le discours épistolaire dans la panoplie de discours produits autour et pendant la GG, et qui par là permettent de passer de l'échange épistolaire au champ discursif où les lettres circulent. La prise en compte de la double dimension de ces discours serait d'ailleurs à la base de toute étude des interactions, soient-elles écrites ou orales, en face à face ou en différé, ainsi que l'écrit Peytard :

\footnotetext{
«[...] dire "interaction verbale", ce n'est pas seulement prendre en compte ce qui, dans le face-à-face d'un individu et d'un autre individu, dans un dialogue, psychologiquement, logiquement et linguistiquement, se produit par concaténation, c'est prioritairement penser l'interaction réalisée dans/par l'ensemble des multiples discours d'une société donnée en un moment donné. Penser que ces discours, dans leur multitude indéfinie, interagissent les uns avec les autres. Et tout duo/dialogue singulier ne peut être analysé hors de l'interaction sociodiscursive. » (Peytard 1995 : 36 cité par Moirand, 2004, en ligne).
} 
La prise en compte des conditions socio-discursives de production des discours des poilus se rend donc nécessaire afin d'expliquer la manière dont le «dialogue écrit » qui s'instaure de par ces lettres ouvre sur les chemins de discours autres. J'essaierai donc de vérifier l'hypothèse que les lettres des soldats se configurent comme un terrain d'observation de tensions discursives actualisant à la fois déterminations dialogales et dialogiques. Ces déterminations rempliraient deux fonctions pragmatiques complémentaires au sein de ces lettres, à savoir celle de créer l'illusion d'un dialogue in praesentia, d'inscrire l'autre dans la même situation d'énonciation du locuteur et celle d'établir avec le destinataire un contact direct, une voie privilégiée de transmission d'informations considérées comme plus conformes au vécu du scripteur et à son expérience au front, bref, de conférer au discours une dimension argumentative plus ou moins évidente.

L'intérêt serait alors de montrer non seulement comment dialogique et dialogal interagissent dans ces énoncés, mais aussi d'identifier les effets pragmatiques que cette connexité produirait en discours, à savoir de réduire la distance physique et symbolique entre les deux locuteurs d'une part et permettre aux scripteurs de prendre des distances par rapport à certains discours «dominants», circulant surtout à l'arrière, d'autre part.

Cela dit, il reste à saisir les lieux de ces tensions exprimant la volonté, voire la nécessité, des scripteurs de faire surgir leur point de vue au milieu de ce qu'on peut considérer comme une véritable jungle d'énoncés et de discours autour de la Grande Guerre et des poilus. S'il est vrai que, d'après Bakhtine (1984 [19201974]), le dialogisme, en tant que notion résidant à la base d'une véritable théorie du discours (Moirand, 2004), concerne à quelques degrés près tout énoncé produit par un énonciateur, certains phénomènes seraient plus aptes que d'autres à en montrer le fonctionnement et les enjeux dont il serait porteur dans un genre de discours donné. Dans le cadre du genre épistolaire qui m’occupe, j'essayerai de vérifier mon hypothèse en montrant que la tentative de réduire la distance passe principalement par le biais de deux phénomènes :

(1) des énoncés au présent à la première personne singulière de l'indicatif et des impératifs à la deuxième personne du singulier qui ne seraient pas reconduisibles à la catégorie de «performatif» des pragmaticiens (Austin, 1991[1962], Searle, 1972 [1969]), bien qu'ils agissent de quelque sorte sur l'autre par l'inscription en discours de la situation d'énonciation et par l'attribution du point de vue du locuteur ;

(2) les marques dialogales et dialogiques permettant aux soldats de créer de véritables simulacres de dialogues avec le destinataire tout en ouvrant sur des discours autres, via des degrés variables de distanciation énonciative.

Si ces deux caractéristiques ne sont pas propres au discours épistolaire des poilus (Siess 2010, Jaubert, 2005), dans ces lettres elles permettent d'observer des phénomènes intéressants en ce qui concerne la frontière entre conversation en face à face et genre épistolaire (Adam, 1997, Kerbrat-Orecchioni, 1998) et participent de la construction discursive d'une dimension argumentative (Amossy, 2010 [2000]) articulée sur les contraintes du genre épistolaire et du champ discursif.

Comme on le verra, cette visée argumentative tend à installer un regard qui se veut à la fois critique et intime sur la guerre, sans pourtant risquer d'être censuré. Sans cette visée on ne peut pas comprendre toute l'épaisseur du discours des combattants qui doivent «bouger» dans un ensemble de discours plus ou moins légitimes et de destinataires plus ou moins souhaités.

\section{Retour à l'intime... de par un bout de papier : rôle et fonctions des lettres d'après les poilus}

La lettre sert avant tout à rassurer les poilus et leurs proches, c'est d'ailleurs ce que l'on peut remarquer à partir des nombreux commentaires portant sur la nécessité d'écrire et de recevoir quelques mots de la part de l'autre : 
Je viens de passer les heures les plus tragiques de mon existence. Le temps me manque pour vous écrire. Je suis sain et sauf et vous embrasse. (Bénard H., le 19/12/1914, p. 73)

Je n'ai guère le temps de vous écrire à présent, aussi je vous écris seulement quelques mots pour vous rassurer. (Marquand A., le 12/03/1917, p. 175)

Deux mots pour vous rassurer car vous devez être en souci. Et pour cause. Mais je vous assure qu'il m'a été matériellement impossible d'écrire avant. (Marquand A., le 26/03/1917, p. 177)

Je te chine un peu, mais c'est pour te faire voir mieux que je t'aime et comment ! (Olivier G., le 02/01/1915, p. 290)

Je comprends que c'est ennuyeux pour toi d'écrire tous les jours mais tu as tort de ne pas m'écrire ça ne serait que dix lignes. Ca me fait tant plaisir. Je dis comme toi, écris un peu mais écris. (Olivier G., le 25/11/1914, p. 218)

La lettre est, indépendamment des contenus, un signe de vie, un objet dont la seule réception vaut à rassurer son destinataire. Au-delà de ce qu'ils écrivent, c'est en effet l'objet même de la lettre qui constitue un message consolant : sa réception confirme le fait que le destinateur/destinataire est encore vivant. La lettre devient alors le « gage d'un futur commun possible » (Vidal-Naquet, 2009 : 220). Il s'ensuit que l'absence de lettre, lors du moment collectif de la réception du courrier, est ressentie comme un évènement inquiétant,

Je suis très surpris de n'avoir rien reçu. Avez-vous reçu ma carte-lettre et ma carte postale ? je suis le seul de la chambrée qui n'ait encore rien reçu et je vous assure que c'est un véritable crève-cœur, lorsque à la distribution des lettres, il ne m'arrive rien. (Marquand A., le 27/12/1914, p. 22)

qui entraîne parfois une reproche de la part du scripteur :

(a) Si tu vois Choquet, souhaite lui le bonjour mais dis lui que ce n'est pas gentil de ne pas m'écrire. Il sait pourtant combien une lettre est bien accueillie. (Olivier G., le 24/11/1914, p. 216)

(b) Ma chère Anine, Tu ne m'as pas écrit hier dimanche pour me raconter votre voyage. Ce n'est pas bien. J'ai attendu de vos nouvelles toute la journée, mais il est si tard que je n'y compte plus. Je vais bien, mais le temps est triste aujourd'hui. Ecrivezmoi dès que vous recevez une lettre de moi, c'est-à-dire tous les deux jours. Je vous embrasse tous. (Bénard H., le 21/06/14, p. 156)

(c) Je viens de te demander pardon de la lettre que je t'ai écrite hier ; je t'ai reproché à tort de ne pas m'écrire tous les jours ou d'avoir la frousse de porter ta lettre à la poste le soir. Aujourd'hui j'ai eu l'immense joie de recevoir trois de tes lettres celles du 2728 et 29 novembre, toutes pleines de belles choses de ma petite maison. J'en suis joyeux, encore plus que d'habitude si c'est possible car, il faut bien te figurer que je suis toujours heureux. (Papillon M., le 03/12/1914, p. 227)

Dans (c), le scripteur, après s'être excusé, se hâte de rassurer sa femme sur son moral, comme cela arrive fréquemment :

Il ne faut pas que je te sois une cause de fatigue pour m'écrire longuement, je patienterai jusqu'à ta guérison, Je ne te demande seulement qu'un petit mot pour me rassurer et c'est tout, car je me sens si loin. [...]. La guerre est là avec toutes ses horreurs, on ne peut vivre qu'en l'attente de jours meilleurs. Tarderont-ils ? Là est le problème. (Marquand A., le 29/09/1917, p. 215)

Rassurer et continuer à espérer dans un futur heureux, loin des horreurs de la guerre, apparaissent dès lors deux thématiques strictement liées dans les lettres, dont la réception devient le gage.

Les lettres constituent également la seule possibilité pour les soldats de se confier, d'exprimer leur malaise et leurs peurs face aux conditions de vie imposées par les tranchées, de garder un contact avec leur vie précédente :

je ne sais pas, mais je crois qu'il nous faudrait une bonne victoire pour remonter les hommes. Les officiers même ne cachent pas qu'ils en ont assez. Si je vous dis tout 


\begin{abstract}
ceci, c'est par besoin de le confier à des amis car j'ai peur de quelque chose. Dieu veuille que je me trompe. (Pensuet M., le 06/08/1915, p. 92)

C'est avec beaucoup de plaisir que j'ai lu ta charmante et longue lettre. Vois-tu, continue à me raconter de ces «niaiseries » comme tu dis, car tu dois bien te douter qu'ici une longue lettre est estimée à sa juste valeur !!!_(Marquand A., le 16/10/1917, p. 219)
\end{abstract}

Peu importe ce que l'on y écrit, l'important c'est d'écrire. Un petit mot, quelques niaiseries suffisent pour « transporter » les soldats loin de la tranchée et tout près de leurs interlocuteurs. Les scripteurs confèrent aux lettres le pouvoir de réduire la distance physique qui les sépare de l'arrière et, par là, de faire revivre des conversations :

Tenez, vos lettres, souvent: je les lis, je me sens causer avec vous, et je me sens retrouver ma vie normale - d'où joie- les rêves de même. L'imagination se représente vivement les objets et jouit de cette représentation. (Tanty E., le 2 décembre 1914, p. 188)

Qu'importe l'absence, puisque nous nous écrivons tous les jours et que tous les jours

je t'analyse ma vie comme si j'étais près de toi. (Olivier G., le 31/10/1914, p. 166)

Résister à la montée des horreurs, prendre une distance vis-à-vis de ces moments angoissants en permettant une fuite, même si elle est seulement mentale, et le « retour » à une dimension intime, autant de fonctions remplies par ces missives.

Les lettres constituent donc, pour les soldats, la seule manière de se créer une place dans le monde et d'inscrire leur propre parole dans le cours des évènements historiques. Elles manifestent une volonté désespérée de contact avec leurs proches, un fort besoin de renouer des relations avec ceux qu'ils ont dû laisser, pour un temps indéfini et au nom d'idéaux incertains et ressentis comme de plus en plus artificiels au fil des années de guerre. C'est ainsi que Trévisan écrit à ce propos : " la lettre apparaît comme une façon de préserver sa singularité, de se déprendre de l'étreinte mortelle du collectif » (Trévisan, 2003 : 332). D'où la surabondance de traces inscrites dans la matérialité discursive aptes à réduire les distances, objective et symbolique, comme les analyses présentées dans les paragraphes suivants le montreront.

\title{
3 Partager la situation d'énonciation à distance
}

Comme l'affirme Kerbrat-Orecchioni (1998), l'une des différences principales entre conversation en face à face et correspondance épistolaire tient au fait que dans l'une les tours de parole sont co-construits en présence dans une situation de communication partagée par deux ou plusieurs interlocuteurs, alors que dans l'autre, il s'agit plutôt de tours de parole plus longs, monogérés par le scripteur et dont le caractère prémédité est évident dans l'absence d'interruptions et de chevauchements. Or, comme l'affirme fort bien Jaubert :

La spécificité de la lettre [...] réside dans le rapport entre l'absence physique de

l'Autre, et sa présence que le discours projette. L'interaction véhiculée s'inscrit

d'abord, et nécessairement, dans ce rapport. (Jaubert, $2005: 225$ )

Pour les lettres, l'enjeu concernerait donc la manière avec laquelle les interlocuteurs gèrent cette distance physique/présence en discours, à savoir les moyens discursifs exploités par les scripteurs afin de créer l'effet de proximité entre eux. Dans les lettres des poilus, la distinction nette entre les deux genres semble en effet s'atténuer si l'on prend en compte des énoncés à la première ou à la deuxième personne du singulier qui, me semble-t-il, permettent de réunir destinateur et destinataire dans la même situation d'énonciation, bien que fictivement :

J'interromps un instant ma lettre pour rentrer à l'intérieur suivant l'ordre, car un aéro apparait. Je ressors [...] (Marquand A., le 07/06/1915, p. 46).

Dans ces énoncés, le scripteur explicite les circonstances extralinguistiques qui seraient visibles lors d'une conversation en face à face : cette situation d'interruption momentanée de l'écriture correspondrait en effet, lors d'une conversation, à un moment de pause, d'interruption des tours de parole, qui n'aurait 
aucun besoin d'être mis en mots. C'est bien là une caractéristique de l'écriture épistolaire quelque peu paradoxale : si, d'un côté, les références au cadre spatio-temporel, c'est-à-dire à ce qui se passe au moment de l'acte de l'écriture ("rentrer à l'intérieur en suivant l'ordre, car un aéro apparait »), seraient superflues lors d'une conversation et, par là, elles ne feraient que souligner l'absence du destinataire, de l'autre, elles permettent d'inscrire la situation d'énonciation dans l'échange à distance.

Dans cette catégorie d'observables, j'ai regroupé tant des énoncés à la première personne du présent de l'indicatif que des énoncés à la deuxième personne singulière de l'impératif car ils permettent d'observer des phénomènes intéressants en ce qui concerne la manière dont les scripteurs essaient de partager avec leur destinataire / faire partager l'acte d'écriture.

A côté des caractéristiques morphosyntaxiques communes (première personne du présent de l'indicatif et deuxième personne de l'impératif), qui à elles seules ne suffiraient pas à cerner une classe d'observables pertinents pour la présente analyse, ces énoncés se caractérisent par le fait que leur inscription en discours interrompt ou, du moins, feint interrompre le récit exposé dans le reste de la lettre. En permettant de passer du récit au discours, ces énoncés opèrent donc de quelque sorte en conférant au discours le même caractère improvisé des échanges oraux.

En particulier, pour ce qui est des énoncés au présent de l'indicatif vus plus haut, ils décrivent des actions quotidiennes, simples, accomplies par le scripteur pendant qu'il écrit et ils permettent d'inclure le destinataire dans le hic et nunc de la conversation. Cet effet de proximité est encore plus évident là où ce genre d'énoncés se combine avec les énoncés à l'impératif, comme dans l'exemple suivant :

J'ai reçu tous les colis qu'on m'a annoncés. Les camarades sont comme moi. Attends, j'allume ma pipe. (Olivier G., le 22/11/1914, p. 212)

Dans ce dernier extrait l'on voit les différents statuts du présent de l'indicatif dans ces lettres et son interaction avec les autres temps et modes. Le premier énoncé «Les camarades sont comme moi » décrit une situation stable dans le temps, c'est le présent de la narration dont l'emploi, tout en actualisant le propos, n'a rien de surprenant dans une lettre : l'emploi du présent est en effet justifié par la nature de l'information donnée, surtout si on la met en perspective avec l'action ponctuelle de la réception des colis, au passé composé. Le deuxième énoncé au présent «j'allume ma pipe » suit l'impératif « Attends » et indique une action en train de se faire. Si l'on ne peut pas considérer ces énoncés comme des performatifs en ce qu'au niveau sémantico-énonciatif leur énonciation ne permet d'accomplir aucune action, on ne peut pas nier qu'ils agissent ou entendent agir sur la perception de la situation d'énonciation du destinataire : les scripteurs font «comme si » (Récanati, 1995) le destinataire était là, partageant l'instant avec eux.

Pendant que je t'écris il faut que je surveille le thé, attends. En fait d'attendre, on est venu me chercher et le thé a été tout bu ! (Pensuet M., le 09/12/1914, p. 237)

C'est le je-ici-maintenant de l'acte d'écriture transposé dans une situation qui, de par sa nature même, implique une distance spatio-temporelle. La présence massive d'embrayeurs personnels et spatiaux qui reproduisent des situations dialogales ne fait que confirmer cette transposition et en exacerbe les effets :

J'ai commencé cette lettre ce matin et j'ai à peine eu le temps de mettre la date, qu'il a fallu mettre sac au dos. Me revoilà. (Olivier G., le 16/11/1914, p. 198)

Le scripteur introduit ici le déictique « revoilà » qui montre jusqu'à quel point l'acte d'écrire est conçu à l'instar d'un véritable rendez-vous avec un bout de papier pour arriver à l'autre ou mieux, pour l'inclure dans sa propre situation d'énonciation. L'emploi du présentatif est révélateur de cette attitude en ce que, comme le soutient Riegel, il « sert à désigner un référent dans la situation d'énonciation » (Riegel et al. 2009 [1994] : 757) et présuppose donc la présence physique du scripteur.

Le fait que les actions introduites par ces énoncés relèvent d'une banalité frappante, à savoir des moments de la vie de tranchée parfaitement négligeables dans un contexte où le but de la communication serait celui d'informer l'autre, de le rassurer, de partager ses pensées et ses émotions, ne fait que confirmer que ces énoncés répondent prioritairement à la volonté d'établir un dialogue plus serré : 


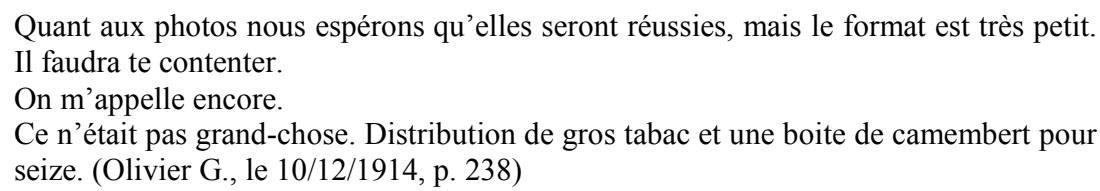

La pause est reproduite au niveau de la structuration du texte par une cassure syntaxique : le scripteur va à la ligne pour introduire dans le dialogue le tiers-absent par l'indéfini on et, par là, ouvrir sur la description des actions presque rituelles et monotones («ce n'était pas grand-chose ») de la vie au front. Le scripteur laisse entendre que ce qui se passe au front a peu d'importance par rapport à l'écriture que ces voix vont troubler. Ce pronom «on » interrompt la conversation et la construction discursive d'une dimension intime pour rappeler au soldat et à sa femme la distance qui les sépare.

Certains commentaires de ce genre portent même sur les conditions matérielles de l'écriture, comme dans l'exemple ci-dessous, où le scripteur explicite le manque d'encre dans son stylo dans une incise entre parenthèses :

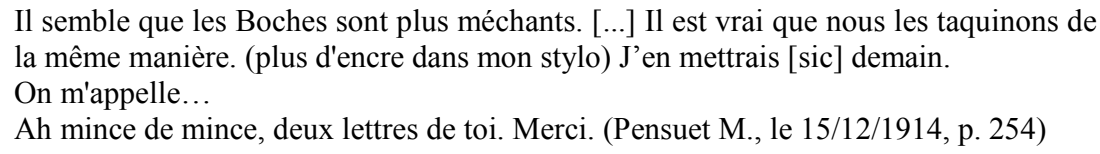

Suite à la référence à l'encre, l'on retrouve encore une fois, sous la plume d'un autre scripteur l'énoncé «on m'appelle» suivi de points de suspension et, enfin, l'interjection qui renforce l'effet d'immédiateté et d'oralité. En particulier «Ah mince de mince » est une interjection propre de l'oral qui trahit la surprise et la réaction non préméditée de la part du scripteur. Il partage ou du moins feint de partager ses émotions pour la réception des lettres avec sa femme au moment où il les éprouve. Ces exemples témoignent du moins de la volonté de forcer certaines contraintes liées au genre et au support choisi ainsi qu'à la double distance, physique et symbolique, imposée aux scripteurs et à leurs proches.

La présence massive de ces énoncés au présent et à l'impératif peut etre interprétée à partir d'une perspective cognitive telle qu'elle est suggérée par Récanati (1995). A la base de ces énoncés résiderait un procédé d'idéalisation métaphorique où la conversation orale est considérée comme le modèle prototypique de l'échange verbal : " on fait comme si l'auteur de la lettre se mettait à 'parler' au destinataire au moment même où celui-ci commence à la lire » (Récanati, 1995 : 43, ital. de l'auteur). Les déictiques et les interjections repérés semblent confirmer le bien-fondé de cette interprétation tout comme la présence d'autres cas de figure qu'on pourrait considérer comme complémentaires aux exemples analysés jusqu'ici, où les scripteurs mettent en scène le moment de la réception de la lettre de la part de leur destinataire :

\begin{abstract}
Te rappelles-tu nos premiers mois de mariage, les as-tu devant les yeux bien réels, biens grands ouverts comme lorsque nous y étions ? Nous nous aimions, mignonne, immensément. J'aime me rappeler ces jours-là. Ce sont les plus beaux d'une vie ! [...] Quand la lettre sera lue je sais que tes yeux se porteront vers les miens et je lirai les mêmes mots que ton cour contient pour moi, et tes yeux me diront : je t'aime. (Olivier Gaston, à sa femme, le 17 octobre 1914, pp. 138-139)
\end{abstract}

Si dans les autres exemples, les scripteurs inscrivaient leur point de vue, leur moment d'énonciation dans la textualité de la lettre, cet extrait montre qu'ils peuvent aussi adopter le point de vue de leurs detinataires et se projeter au moment de la réception. Le futur employé pour la description de cette scène manifeste le changement de point de vue du scripteur qui attribue à son destinataire même des gestes, des regards, des états d'âme, voire des mots non prononcés («tes yeux me diront : je t'aime »). Pour le dire avec Récanati (2008), par rapport au premier paragraphe de l'extrait au passé, au niveau pragmatique, il y a bien là un changement de «contexte interne » du point de vue du scripteur : du contexte réel, où la parole se produit effectivement, il passe à un contexte interne «où l'on prétend qu'elle [la parole] se produit» (Récanati, 2008: 2). Les scripteurs semblent donc par là exploiter les potentialités que l'échange épistolaire permet en termes de changements de points de vue et, par là, de reconfiguration des 
rapports de place entre eux et leurs destinataires. Que les échanges soient envisagés au moment présent ou projetés dans un futur plus ou moins lointain, ce sont les intentions communicatives des scripteurs qui priment dans ces lettres sur le contexte réel de l'énonciation. Or, comme le soutient Jaubert :

Certes, il y a bien échange, c'est-à-dire interaction, mais cette interaction se développe in absentia, elle permet à la fois de se situer dans le prolongement d'une interaction en face à face, et de la reconfigurer sans les astreintes de la coopération conversationnelle, sans les interventions réactives (parasitantes, inhibantes ou encourageantes) de l'autre, et donc selon les voeux et le rythme du locuteur qui a pris la plume. Dès lors elle répond à une détermination plus dialogique que dialogale. (Jaubert, $2005: 216$, ital. de l'auteure)

Ce sont justement les déterminations dialogales et dialogiques caractérisant les lettres des poilus et dont l'orientation confère aux textes pris en compte une dimension argumentative toute particulière qui seront analysées dans le paragraphe suivant.

\title{
4 Enoncés dialogiques, dialogaux et dimension argumentative.
}

Les lettres, on le sait, sont saturées de formes de dialogisme et de dialogue (Adam, 1997, KerbratOrecchioni, 1998, Jaubert, 2005) : elles suppléent l'absence de l'autre et permettent aux interlocuteurs de se projeter dans un dialogue fictif avec cet autre. Or, si cela est une caractéristique du macro-genre épistolaire,

La pratique épistolaire peut se diversifier en autant de sous-genres que l'on imagine de types d'interactions entre des individus. Lieu de rencontre de plusieurs dialogisations, imbriquées comme on l'a vu, la lettre les actualisera à des degrés, et sous des formes variables, en fonction de l'interaction qu'elle véhicule. (Jaubert, $2005: 225)$

Je voudrais montrer que, dans le cas des lettres des poilus, cette surabondance de formes dialogiques contribue non seulement à créer un simulacre de dialogue, mais aussi à installer en discours une dimension argumentative (Amossy, 2010 [2000]) qui répond, comme le dit Housiel, à la nécessité de « transmettre une expérience » (Housiel, 2012, en ligne).

Il s'agit là d'une expérience que les destinataires ne connaissent point et que les destinateurs transmettent, mais toujours à partir d'un point de vue critique. Cette dimension argumentative ne peut être appréhendée qu'à travers la prise en compte du champ discursif dans lequel les mots des poilus se situent, à savoir les discours autres tenus sur la guerre elle-même et dont les scripteurs veulent se distancier de manière plus ou moins cachée. C'est du moins ce que l'on peut comprendre à partir de l'exemple suivant, où le scripteur thématise la situation particulière où l'échange épistolaire pendant la guerre a lieu :

\begin{abstract}
La censure, tu le sais, est impitoyable ici et certains pauvres poilus ont appris à leurs dépens qu'ils ne devaient pas avoir la langue trop longue, ni même recevoir des lettres (qui sont d'ailleurs supprimées) sur lesquelles les parents ont souvent aussi la langue un peu longue. C'est révoltant mais c'est ainsi. Il semblerait qu'une lettre est une chose sacrée, il n'en est rien. Sois donc prudente, ma chérie, et si tu veux que je reçoive toutes tes lettres, ne me parle pas de la guerre. Contente-toi de notre grand amours [sic], cela vaut beaucoup plus que tout. (Bouvard H., le 03/12/1917, p. 92)
\end{abstract}

$\mathrm{Si}$, comme on l'a vu au $\S 3$, l'écriture dans les tranchées répond à la volonté de tisser des liens, d'exprimer un point de vue intime sur la vie au front, l'expression de ce même point de vue peut représenter la raison d'une sanction de la part des censeurs. Lorsqu'ils écrivent, les poilus savent bien que le destinataire auquel ils s'adressent n'est pas le seul lecteur de leurs textes : l'autorité militaire par le biais de l'intensification du contrôle postal (Saint-Fuscien, 2011) atteint un niveau de capillarité remarquable afin de limiter tout passage dangereux d'informations et tout genre de discours de contestation de la guerre et des actions militaires. L'on est donc en droit de s'attendre à ce que le discours critique passe principalement par une représentation particulière que le discours des poilus donne de ses 
rapports à l'autre par le biais de différents degrés de distanciation énonciative tout en installant en discours une dimension argumentative apte à emporter l'adhésion du destinataire.

Je partirai des marques les plus récurrentes et, en même temps, les plus neutres du point de vue argumentatif, pour passer enfin au crible de l'analyse des extraits caractérisant de plus près ce type de correspondance. Il est fréquent de repérer des cas de reprise directe des mots que le destinataire a adressés au soldat dans une lettre précédente. Dans ces cas, c'est la dimension dialogale qui prime et qui garantit le suivi thématique d'une lettre à l'autre, comme dans les extraits suivants :

(a) $\mathrm{Si}$ je me souviens, ce matin je te disais que je mangeais bien (Pensuet, M., le

11/10/1914, p.129)

(b) D'ailleurs nous sommes très bien nourris comme j'ai dû te le dire (Pensuet, M., le

$11 / 10 / 1914$, p.130)

(c) Tu me dis aussi dans la même lettre que tu vas mieux. Tu ne m'avais jamais dit que tu étais malade. (Papillon M., le 31/10/1914, p.130)

(d) Dans ta lettre tu me demandes si je n'ai plus besoin de ceci, de cela. Non. (Marquand A., le 27/06/1915, p.147)

(e) Dans ta lettre d'aujourd'hui tu me dis que je sais pourquoi tu n'as pas envoyé le briquet. (Olivier G., 13/03/1916, p.147)

(f) Je t'ai parlé de nos chambres à coucher dans la terre, Nous continuons toujours à en faire et un peu à la fois on arrive à en faire des épatantes, on dirait des fosses à purin ! $\mathrm{Si}$ tu pouvais voir ce pays ravagé. (p. 155)

Ces exemples remplissent la fonction principale de tisser la conversation en différé, de mettre en relation les lettres, l'une après l'autre. On remarque d'ailleurs que ce sont les verba dicendi dire, demander et parler qui sont utilisés pour rappeler son propre discours et le discours de l'autre. Si l'on regarde de plus près les contenus introduits par ces marques, l'on s'aperçoit qu'elles introduisent soit des mots pour rassurer l'autre (de (a) à (e)) soit des reprises de mots ou expressions susceptibles de représenter de très près la vie des tranchées et, par là, d'entraver la bonne compréhension de ce qu'on a dit dans les lettres précédentes (f).

Il arrive pourtant que la reprise de conversations précédentes réponde à la nécessité de prendre des distances par rapport à ce qui a été écrit par l'autre tout en lui permettant de partager sa propre expérience de la guerre :

Dans ta dernière lettre tu me parles de mes quatre jours de repos. Ça m'a fait rire tout de même. Si tu voyais ce que c'est que notre repos !!! [...] Ces repos consistent à creuser des abris de cinq à six mètres de profondeur sur huit et quatre de long et large, puis à abattre des arbres et à faire un tas de choses qu'il serait trop long de te dire. (p. 237)

Dans les tranchées les mots décrivant la vie des soldats se chargent de nouvelles significations, charrient d'autres connotations par rapport aux mêmes mots utilisés avant la guerre et/ou par les civils : les reformuler, les spécifier et les commenter font donc surgir au fil du discours une réflexivité permettant de réduire la distance, même linguistique, ressentie par les soldats comme incommensurable (« il serait trop long de te dire »), et à laquelle seule la vue directe pourrait constituer une solution valable (« Si tu voyais ce que c'est que notre repos !!! », et cela vaut également pour le commentaire (f)). Le scripteur montre à l'autre la réalité de la guerre, comme si celui-ci pouvait la voir de ses propres yeux. L'on remarque que cette fois-ci « voir » est introduit dans une exclamation renforcée par une ponctuation de type émotionnel, contribuant à emporter l'adhésion pathémique/pathétique du destinataire et donc à partager avec lui le même état d'âme. C'est justement cette nécessité d'aller au-delà de la distance, qui, me semble-t-il, justifie la présence de verbes constatifs et d'attitude propositionnelle qui fonctionnent à l'instar d'embrayeurs par lesquels les scripteurs attribuent des dires, des sensations et des pensées à leurs interlocuteurs :

Tu vois que dans le métier (fichu métier) il y a du bon quand même. (Papillon M., le 12/09/1915, p. 164) 
L'énoncé «tu vois» peut être en effet reformulé en «tu ne peux que constater avec moi » tout en inscrivant par la deixis du sémantisme du verbe «voir » l'interlocuteur dans la situation d'énonciation. A remarquer également la présence de l'adverbe " quand même » à la fin de l'énoncé, qui montre la volonté du poilu de reconfigurer positivement la représentation du métier de soldats que son destinataire est censé avoir formée d'après, sans doute, les récits du scripteur lui-même : il s'agit bien là d'un indice de dialogisme ouvrant sur le chemin de discours antérieurs et dont l'origine énonciative n'est pas certaine. Ce n'est pas le cas de l'extrait suivant, où la citation directe des mots du destinataire constitue le départ de l'argumentation plutôt eplicite du scripteur :

\begin{abstract}
là où tu me traites carrément "d'ivrogne" ou que je mène "une vie d'orgie". Voilà tes propres termes! [...] Puisque comme tu le dis toi-meme, on ne peut trouver actuellement ce que l'on pouvait se payer en temps de paix (là je te comprends fort bien), il est bien juste qu'on s'amuse et qu'on essaye de s'amuser comme l'on peut (Anonyme, p. 153)
\end{abstract}

La reprise entre guillemets des insultes ne répond pas ici à la seule volonté de tisser les fils de la conversation écrite et à distance, mais surtout à manifester explicitement, par la suite, son désaccord avec le jugement considéré comme trop sévère. Les dires sont alors cités pour permettre au scripteur de s'en détacher. Dans la suite, les mots du destinataire sont en effet repris, cette fois-ci sous la forme de simple mention, pour supporter les dires du scripteur et, par là, justifier la nécessité de s'amuser « comme l'on peut ». Par la glose métadiscursive «comme tu le dis toi-même » le scripteur se réapproprie le point de vue de son interlocuteur et l'utilise en guise d'appui de ses propres dires.

Ce genre d'énoncés fonctionne donc tant du côté de la construction de la dimension argumentative que de la création du simulacre de dialogue, tout comme les marques dialogales suivantes, par lesquelles s’instaure un « jeu » énonciatif de projections des discours entre destinateur et destinataire :

\begin{abstract}
(a) Vous allez dire que ma lettre n'est pas encourageante. Que voulez-vous, je suis franc et j'ai tenu à vous dire tout cela. Et encore : que tous les journaux ne font que monter les hommes car ils sont en contradiction avec tout ce que nous voyons. Ils nous montrent les civils allemands crevant de faim! Comment se fait-il que si l'on prend $10 \mathrm{~m}$ de tranchées et que l'on trouve des sacs, tous contiennent saucisson, jambon, beurre, etc. Ils n'ont pas de pain, mais du riz au lait épatant et à pleins bouteillons. Et l'on vient nous dire qu'ils demandent grâce. (Pensuet M., le 29/07/1915, p. 86)

(b) vous allez me dire encore que je ne suis pas encourageant en ce moment, excusezmoi mais quand je me suis soulagé je supporte mieux les mille tracasseries du métier. Et combien de choses encore que l'on voit, que l'on sait, que l'on ne dit pas ! (Pensuet M., le 01/02/1916, p. 151)
\end{abstract}

Les destinateurs attribuent aux destinataires des énoncés, des pensées, des sentiments. Si d'un côté cela crée un dialogue fictif et, par conséquent, un rapprochement entre les deux scripteurs, de l'autre, il permet au soldat d'inscrire une dimension argumentative visant la critique de la guerre : c'est ainsi que ces simulations de dialogues portent sur le découragement du soldat que les journaux ne semblent pas prendre en compte (dans (a)) ou dont eux-mêmes ne parlent pas beaucoup (dans (b)) en raison de la censure ou de l'autocensure. Le premier extrait est intéressant en ce que plusieurs phénomènes de nature dialogique et dialogale sont imbriqués. D'entrée des jeux, le scripteur inscrit en discours ce qu'il prétend présenter comme les mots de son destinataire en guise d'anticipation de la réponse attendue à ce qu'il vient de décrire. Il fait ensuite «dialoguer» ces dires avec «Que voulez-vous, je suis franc », où l'affirmation péremptoire de sa franchise anticipe l'expression de sa méfiance par rapport au discours de la presse, présentés comme peu conformes à la réalité. Le scripteur non seulement inscrit par là en discours un simulacre de dialogue, mais aussi il prend ses distances des discours autres, identifiés dans ceux tenus par les journaux. «Je suis franc» entretiendrait alors des relations dialogales et dialogiques à la fois avec l'environnement discursif qui précède et celui qui suit son énonciation par la prise de distance par rapport à ces autres discours.

D'autres simulations traitent de la description des moments de l'expérience guerrière que les destinataires ne pouvaient pas connaître si ce n'est qu'à travers l'expérience de ces lettres : 
Vous m'objecterez aussi : « et ton prêt? ». Mais je vous répondrai, en «baissant la tête », que presque la totalité passe dans la popote. (Marquand, A., le 17/04/1917, p. 205)

L'attribution d'une question directe au destinataire renforce non seulement l'effet dialogal en projetant les deux interlocuteurs dans un futur plus ou moins lointain de par l'emploi du futur simple, mais elle anticipe sur des énoncés qui pourraient être produits par l'énonciateur lui-meme («je vous répondrai... » et par son destinataire « et ton prêt ? ») en affichant par là la double caractérisation dialogale et dialogique de cette séquence. Le scripteur prévient de quelque sorte une objection possible si l'on ne connait pas ce qui se passe au front. Ce genre de situation peut se retrouver également là où les scripteurs ressentent le besoin de revenir sur leurs mots afin d'en expliciter le sens :

Tu t'es peut-etre demandé ce que c'était que bombardier. C'est tout simplement le soldat qui prépare la poudre, la mèche et qui met la bombe dans le mortier (Olivier G., le $01 / 11 / 1915$, p. 255)

La simulation de dialogue enchaîne sur l'introduction d'une glose définitionnelle d'un terme spécialisé, susceptible de ne pas être transparent pour le destinataire. Cet extrait montre comment réflexivité métalinguistique et marques dialogales peuvent se conjuguer dans ces lettres afin d'éliminer la distance physique, mais aussi linguistique, qui semble augmenter au fil des années de guerre entre les deux univers du front et de l'arrière. Dans cet ordre d'idées, certaines simulations montrent la conséquence ultime de cette incommunicabilité, à savoir le silence dit et montré :

Tu me demandes ce que je pense de la guerre. Je ne puis rien te dire de plus que ce que je t'ai dit. Pour que cela aille vite, il faut que les Allemands soient hors de France et de Belgique (Marquand A., le 04/03/1917, p. 293)

Je ne vois plus rien à te dire par écrit, Tu penses et tu devines toutes les bonnes paroles que je te dirais si tu étais là. (Olivier G., 18/08/1916, p.111)

et la dénonciation du décalage entre les mots et les realia:

Tu vas croire que j'exagère, non. C'est encore en dessous de la vérité. On se demande comment il se peut que l'on laisse se produire de pareilles choses. Je ne devrais peutetre pas décrire ces atrocités, mais il faut qu'on sache, on ignore la vérité trop brutale. (Pigeard René, caporal, le 27/08/1916, p. 54)

Ce dernier exemple montre dès le début une simulation de dialogue reposant sur l'idée que le poilu a à propos de ce que son père sait ou croit de la guerre et de la vie aux tranchées. L'inscription de ce dialogue fictif permet au scripteur non seulement de refuser une objection possible, mais aussi de préparer son père à la lecture d'une vérité qui peut paraître invraisemblable aux yeux d'un civil et de créer un effet de proximité et de connivence avec lui, avec qui il semble dialoguer de vive voix. Dans la suite, le scripteur n'entre pas dans le détail des réalités inimaginables en se limitant à y faire des allusions par les hyperonymes «choses » et «atrocités » où la connotation négative dans l'un est assurée par l'adjectif « pareilles » et dans l'autre par la sémantique même du substantif. L'on remarque d'ailleurs que dans «pareilles choses» et «ces atrocités» les premiers éléments des syntagmes ne renvoient pas à des éléments contextuels, se situant avant ou après dans le texte de la lettre : cela rapproche du moins en partie leur fonctionnement discursif à celui des déictiques propres à une situation de communication in praesentia et fonctionnent comme des déictiques montrant directement ce à quoi ils se réfèrent.

De par ces énoncés, les scripteurs mettent en évidence jusqu'à quel point leur expérience constitue un évènement extraordinaire : ils exhibent une impossibilité de dire justement pour dire plus que ce qu'ils voudraient ou devraient. Ils attribuent à leurs proches l'impossibilité de croire à certaines réalités vécues par les soldats comme particulièrement surprenantes: déterminations dialogales et dialogiques se combinent de manière à ce que les scripteurs projettent sur l'autre leurs idées, pensées et perceptions. Ceux-ci recherchent par là une communion de sentiments et d'états d'âme, un sentir avec l'autre, tout en installant une dimension critique de leur vécu et des discours officiels et dont la portée est atténuée par le fait de passer par la bouche de leurs interlocuteurs. 


\section{Remarques conclusives}

Comme on l'a vu, les scripteurs exacerbent le pouvoir communicatif et sentimental des lettres. Ils exploitent toute l'ambigüité intrinsèque au genre épistolaire qui se joue dans le rapport entre l'absence physique et la présence de l'autre en discours ainsi que la possibilité, de par les lettres, de faire surgir des conversations idéales, imaginaires avec l'autre. Aux lettres est ainsi conférée la faculté d'annihiler idéalement les distances, physique et symbolique, grâce auxquelles cependant elles trouvent leur raison d'exister. En leur attribuant des propos et des états d'âme, les scripteurs élèvent au rang de témoins de l'expérience guerrière les destinataires, au même titre que les poilus. Les poilus leur permettraient ansi de voir les mêmes réalités déroutantes et d'éprouver les mêmes émotions pour, enfin, en prendre les distances.

En effet, si les marques prises en compte ne sont pas exclusives des correspondances des années 14-18, l'on assiste bien là à un phénomène tout particulier par lequel les marques dialogiques et dialogales remplissent deux fonctions différentes mais complémentaires au sein des lettres analysées : d'un côté, elles contribuent à créer ce qu'on peut appeler un simulacre de dialogue, où les deux scripteurs semblent partager la même situation d'énonciation, de l'autre elles permettent d'inscrire en discours une prise de distance subreptice par rapport aux discours officiels, dominants dans le champs discursif constitué des discours circulant autour et pendant la GG. Dans ces lettres l'on voit bien comment écrire, c'est aussi agir sur le destinataire pour en modifier le point de vue et reconfigurer les rapports de force entre scripteur, destinataire et champ discursif : dans la simple transmission d'informations du front se glisse la volonté de se détacher de certains discours tenus sur la guerre, mais ressentis comme dépourvus de sens, fondamentalement faux, par les soldats. Les exemples analysés montrent en effet combien ces lettres permettent de reconfigurer les rapports de force dans ce qu'on peut considérer comme une véritable bataille des dires à l'intérieur du champ discursif où elles circulent.

Les obervables analysés mettent donc bien en lumière les « rapports de place » qui se dessinent au fil des discours de cette "parole intermédiaire» (Siess, Hutin, 2005) qu'est la lettre au sein d'une correspondance : les scripteurs mettent en scène leur propre image des destinataires, ils créent leurs interlocuteurs idéaux, censés partager leur même point de vue sur la guerre et sur la vie quotidienne au front. Ils négocient les places respectives, ils reconfigurent constamment leurs dires et les dires de l'autre en les adaptant, en les reprenant au fil du discours et en s'en détachant parfois. S'il s'agit bien évidemment d'une construction fictive, discursive, il n'en demeure pas moins qu'elle semble répondre davantage à une nécessité qu'à une volonté délibérée de manipuler le point de vue de l'autre pour l'englober dans celui du scripteur. Ce qui prédomine c'est, me semble-t-il, la nécessité d'établir un contact le plus direct et le plus sincère possible avec son destinataire, un lien de paroles, comme s'il se tenait de vive voix, tout en créant une voie de transmission des informations privilégiées, qui fasse l'empasse des discours officiels, en dépit des obstacles représentés par la censure, l'autocensure et par les lacunes lexicales. Et encore, cette nécessité peut etre appréhendée par le biais de phénomènes de distanciation énonciative plus ou moins évidents le long des textes et qui permettent d'ouvrir sur des discours autres tout aussi que sur ceux des interlocuteurs.

Le caractère fictif de ce que l'on peut considérer comme une véritable illusion se révèle avec toute sa force au terme du conflit, lorsque les poilus rentrent chez eux (Horne, 2005) : la proximité physique et la rencontre avec l'autre si attendue révèlent en effet toute la difficulté à rendre compte de l'expérience guerrière et les soldats semblent être condamnés à l'incomunicabilité.

\section{Corpus}

Bénard, H.(1999). De la mort, de la boue, du sang. Lettres de guerre d'un fantassin de 14-18, Paris : Grancher.

Foch, F. (éd.) (1922). La dernière lettre écrite par des soldats français tombés au champ d'honneur 1914-1918. Paris : Flammarion.

Guéno, J.-P. (éd.) (1998). Paroles de Poilus Lettres et carnets du front de 1914 - 1918. Paris : Librio. 
Marquand, A. (2011). Et le temps, à nous, est compté" : Lettres de guerre (1914-1919). Forcalquier : C'est-à-dire.

Olivier, G. (2008). Afin de ne jamais oublier, Vie et mort d'un poilu héroïquement ordinaire, Gaston Olivier, soldat au 274e RI. Le Chaufour : Editions Anovi.

Papillon, L. (2003). «Si je reviens comme je l'espère ». Lettres du Front et de l'Arrière. 1914-1918. Paris : Grasset.

Pensuet, M. (2010). Écrit du front, Lettres de Maurice Pensuet, 1915-1917. Paris : Tallandier.

Tanty, E. (2002). Les violettes des tranchées, Lettres d'un Poilu qui n'aimait pas la guerre. Paris : Editions Italiques.

\section{Références bibliographiques}

Adam, J.-M. (1997). Les genres de discours épistolaire. De la rhétorique à l'analyse pragmatique des pratiques discursives. Pratiques, 94, 37-53.

Adam, J.-M. (2006 [2005]). La linguistique textuelle. Introduction à l'analyse textuelle des discours. Paris : Armand Colin.

Amossy, R. (2010 [2000]). L'argumentation dans le discours. Paris : Armand Colin.

Austin, J. (1991[1962]). Quand dire, c'est faire. Paris : Seuil.

Authier-Revuz, J. (1995). Ces mots qui ne vont pas de soi. Boucles réflexives et non-coïncidences du dire. 2 t. Paris : Larousse.

Bakhtine, M. M. (1984 [1920-1974]). Esthétique de la création verbale. Paris : Gallimard.

Branca-Rosoff, S. (1990). Conventions d'écriture dans la correspondance des soldats. Mots. Les langages du politique, 24, 21-35.

Bres, J. (2005). Savoir de quoi on parle : dialogue, dialogal, dialogique ; dialogisme, polyphonie.... In Bres J. (éd.), Dialogisme et polyphonie. Approches linguistiques. Bruxelles : De Boeck Université.

Caffarena, F. (2007). Le scritture dei soldati semplici. In Gibelli A. (sous la dir.). La prima guerra mondiale. Edizione italiana. Torino : Einaudi, t 2, 633-647.

Cazals, R., Rousseau, F. (2001). 14-18, le cri d'une génération. Toulouse : Privat.

Horne, J. (2005). Entre expérience et mémoire : les soldats français de la grande guerre. Annales. Histoire, Sciences sociales, 5 , 903-919.

Housiel, S. (2012). Le discours épistolaire de la Grande Guerre : images et témoignages. Villeneuve, J. (éd.). L'Expérience de la guerre entre écriture et image. Montréal: Département d'Études littéraires. [En ligne. Consulté le 29/11/2013. http://experiencedelaguerreecritureimage.uqam.ca/ Resumes/resHousiel.html]

Jaubert, A. (2005). Dialogisme et interaction épistolaire. In Bres J. (éd.), Dialogisme et polyphonie. Approches linguistiques. Bruxelles : De Boeck Université, 215-230.

Kerbrat-Orecchioni, C. (1998). L'interaction épistolaire. In Siess J. (sous la dir.) La lettre entre réel et fiction. Sedes : Paris. [En ligne. Consulté http://humanities.tau.ac.il/adarr/images/L_Linteraction_\%C3\%A9pistolaire_L.pdf]

Kerbrat-Orecchioni, C. (1990). Les interactions verbales. t. I, Paris : Armand Colin.

Moirand, S. (2004). Le dialogisme, entre Le dialogisme, entre problématiques énonciatives et théories discursives. Cahiers de praxématique, $43,189-220$.

Rabatel, A. (2001). La valeur de "on" pronom indéfini/pronom personnel dans les perceptions représentées. L'information grammaticale, $88,28-32$.

Rabatel, A. (2004). L'effacement énonciatif dans les discours rapportés et ses effets pragmatiques. Langages, 156, 317.

Récanati F. (2008). D'un contexte à l'autre. Cahiers Chronos, 20, 1-14.

Récanati F. (1995). Le présente épistolaire : une perspective cognitive. L'Information Grammaticale, 66, 38-44. 
Riegel, M., et al. (2009 [1994]). Grammaire méthodique du français. Paris : Puf.

Saint-Fuscien, E. (2011). A vos ordres ? La relation d'autorité dans l'armée française de la Grande Guerre. Paris : EHESS.

Searle, J. (1972 [1969]). Les actes de langage. Paris : Hermann.

Siess, J. (2010). Introduction. Argumentation \& Analyse du discours, 5. [En ligne. Consulté le 15/12/2013. http://aad.revues.org/1001].

Siess, J. (2005). Présentation. Semen, 20. [En ligne. Consulté le 15/12/2013. http://semen.revues.org/1073].

Trévisan, C. (2003). Lettres de guerre. Revue d'histoire littéraire de la France, 103, 331-341.

Vidal-naquet, C. (2009). Imaginer le retour. L'anticipation des retrouvailles chez les couples pendant la Grande Guerre. In Cabanes B., Piketty, G.(éds.). Retour à l'intime au sortir de la guerre. Paris : Tallandier, 215- 228.

\footnotetext{
${ }^{1}$ Trévisan, C. (2003). Voir Caffarena, F. (2007) pour les chiffres dans les autres Pays européens.

${ }^{2}$ En France, voir le groupe de chercheurs coordonnés par Cazals à Toulouse et par Becker à Péronne.

${ }^{3}$ Saint-Fuscien, E. (2011).

${ }^{4}$ Dans le corpus analysé, les deux seuls recueils où les éditeurs n'ont apporté aucune correction est celui de Papillon et celui de Tanty.

${ }^{5}$ Les parties des exemples commentées ans le texte sont en italique. Mes ajouts entre crochets.
} 